\title{
El derecho de participación en las políticas sociales: el proceso participativo para la elaboración de la Ley valenciana de Servicios Sociales
}

\author{
Glòria Maria Caravantes López de Lerma ${ }^{1}$
}

Recibido: 21 de septiembre de 2019 / Revisado: 08 de octubre de 2019 / Aceptado: 12 de noviembre de 2019.

Resumen. El derecho de participación ciudadana en la configuración de las políticas sociales es un elemento esencial en las nuevas formas de gobernanza y en el ejercicio de la gobernabilidad de los estados democráticos. En el siguiente artículo, se presenta el ejemplo de la Ley Valenciana de Servicios Sociales, desgranando las diferentes fases del proceso de elaboración y profundizando en las aportaciones de los distintos actores sociales. Dicho proceso, comprendido entre el periodo 2015-2019, constó de cuatro etapas con una triple finalidad: 1) hacer partícipes a la pluralidad de actores (ciudadanía, profesionales, entidades locales, entidades del tercer sector, personas expertas) y partidos políticos a lo largo de su elaboración, apostando por el derecho de participación ciudadana como elemento central; 2) desarrollar un proyecto compartido, común y plural acorde a las necesidades y demandas ciudadanas; y 3) sentar las bases de una iniciativa legislativa con los principios de la democracia. Para ello, en el año 2015 la Vicepresidencia i Conselleria d'Igualtat i Polítiques Inclusives apostó por profesionales de Trabajo Social de reconocida trayectoria y experiencia en el ámbito de los servicios sociales al frente del equipo directivo.

Palabras clave: Trabajo Social; Acción Política; Servicios Sociales; Comunitat Valenciana; Participación.

\section{[en] The right to participation in social policies: the participative process of developing the Valencian Law on Social Services}

\begin{abstract}
The right of citizens to participate in shaping social policy is an essential element in the new forms of governance and in the exercise of governability of democratic rule-of-law States. This article presents the example of the Valencian Law on Social Services, specifying the different phases of the development process and examining the contributions from various social actors. The process covered the period from 2015 to 2019 and was carried out over four stages with a triple objective: 1) to secure participation from the range of actors (citizens, professionals, local entities, third-sector entities, experts) and political parties throughout the development process, relying on the right to citizen participation as a central element; 2) to develop a shared, common and plural bill in line with the needs and demands of citizens; and 3) to establish the basis for a legislative initiative founded on the principles of democracy. The Deputy Presidency and Council for Equality and Inclusive Policy (Vicepresidencia i Conselleria d'Igualtat i Politiques Inclusives) selected social work professionals with recognised backgrounds and experience in the area of social services to head the leadership group for this purpose in 2015.
\end{abstract}

Keywords: social work; political action; social services; Valencian Community; participation.

Sumario: Introducción. 1. Los procesos participativos en las políticas públicas: ventajas, retos y dificultades de futuro. 2. El punto de partida en la Comunitat Valenciana: los antecedentes al nuevo modelo social valenciano. 3. Proceso participativo de la Ley Valenciana de Servicios Sociales. 3.1 Primera etapa: de octubre de 2015 a mayo de 2016.3 .2 Segunda etapa: de diciembre de 2016 a noviembre de 2017. 3.3. Tercera etapa: de noviembre de 2017 a enero de 2018. 3.4. Cuarta etapa: de enero de 2018 a febrero de 2019. 4. El derecho de participación ciudadana en los servicios sociales: una cuestión a desarrollar. 5. Conclusiones principales. 6. Referencias bibliográficas.

Cómo citar: Caravantes López de Lerma, G. M. (2020) El derecho de participación en las políticas sociales: el proceso participativo para la elaboración de la Ley valenciana de Servicios Sociales, en Cuad. trab. soc. 33(1), 99-113.

\footnotetext{
Universitat de Valencia. España.

gloria.lerma@hotmail.es
} 


\section{Introducción ${ }^{2}$}

En los últimos años, España ha vivido importantes transformaciones políticas y económicas que han tenido una influencia directa en la configuración y el desarrollo de los derechos sociales fundamentales. De este modo, las nuevas formas de gobernanza han derivado en nuevas respuestas del ámbito político a las necesidades de la ciudadanía. La disciplina del Trabajo Social junto con otras disciplinas afines han contribuido notablemente desde su nacimiento al desarrollo y garantía de los derechos sociales (Barranco, 2009).

Estas formas de gobernanza parten de la premisa que "el público se convierta en actor de los procesos de formulación de políticas" (Canto Chac, 2008, p.11). Para ello, se fomentan mecanismos que promueven la participación ciudadana en la gestión pública, a favor de la transformación de la propia Administración y con mayor grado de accesibilidad para la ciudadanía; es decir, "un nuevo modelo de gestión pública que busca una creciente implicación de los ciudadanos en la acción de gobierno mediante el refuerzo y la educación de una ciudadanía activa, informada, participativa y corresponsable" (Arnáez Arce, 2012, p. 190). La gobernanza se presenta como el método principal para hacer frente a los grandes retos territoriales y urbanos, haciendo especial hincapié en el protagonismo de la sociedad civil y el buen gobierno (Romero y Farinós, 2008; 2011). Por ello, coincidimos con Garcés Sanagustín (2011) en señalar que: "cualquier avance en la participación se identifica inmediatamente con un avance del sistema democrático" (p. 124).

En el ámbito que nos ocupa, en la década de los 1990, Doyal y Gough (1994) señalaban la necesidad de considerar la participación ciudadana en lo concerniente a la satisfacción de las necesidades, destacando que sin la movilización de "la ciudadanía y la acción del Estado, cualquier intento de mejora de la satisfacción de las necesidades de la mayoría de las personas se topará con la hostilidad de los intereses patronales que defienden objetivos sectoriales" (pp. 354 - 355). En lo concerniente al sistema de servicios sociales, el derecho a la participación de la ciudadanía, los recursos técnicos y la Administración: "es a lo máximo que se puede aspirar, y aún esta aspiración parece de difícil alcance por el momento, tanto en nuestro sistema de servicios sociales como en el conjunto de las políticas públicas españolas" (Martínez-Martínez, 2017, p. 123).

A pesar de existir cierta prevalencia en el diseño vertical de políticas públicas que tratan de atajar problemas públicos (Berrios Navarra y Zapata, 2015), a lo largo del presente artículo se presenta la comunidad como sujeto político con derecho de participación y como espacio en el que confluyen personas, fenómenos sociales, recursos, instituciones y profesionales. La relevancia de la comunidad en la acción política reside en hacer frente a los fenómenos sociales con una base de ciudadanía y en el territorio que emanan los mismos. En el caso de la Comunitat Valenciana se ha contado con la comunidad (ciudadanía, agentes sociales, recursos técnicos y Administración Pública) como elemento esencial y protagónico tanto en el proceso de elaboración como en el posterior resultado, materializado en la Ley Valenciana de Servicios Sociales.

\section{Los procesos participativos en las políticas públicas: ventajas, retos y dificultades de futuro}

Desde finales del siglo XX, los movimientos sociales defendieron un nuevo modelo de democracia donde la participación era el elemento nuclear de las formas de gobernanza (Della Porta, 2013), con la capacidad no únicamente de incidir en los poderes públicos, sino también con la capacidad de disentir en la materia (Subirats, 2016).

El derecho a la participación en el ámbito de las políticas públicas obliga necesariamente a replantear los mecanismos, instrumentos y relaciones de poder existentes en nuestras democracias. En medio de este escenario, las formas de gobernanza: "deberán incorporar la capacidad de implicación y decisión directa de la ciudadanía, no solo en los procesos de implementación de las políticas urbanas, sino, sobre todo, en la definición del problema y en la construcción de alternativas" (p. 95). Por ello, en este punto cabe preguntarse: ¿por qué apostar por procesos

Las primeras aportaciones del presente artículo fueron presentadas al VII Congreso de la Red Española de Política Social (REPS, 2018) en Zaragoza bajo el título: "El proceso participativo para la elaboración de la nueva Ley de Servicios Sociales de la Comunitat Valenciana desde el Trabajo Social". 
participativos en la configuración de políticas públicas? En el caso de la Comunitat Valenciana se ha contado con la comunidad (ciudadanía, agentes sociales, recursos técnicos y Administración Pública) como elemento esencial y protagónico tanto en el proceso de elaboración como en el posterior resultado, materializado en la Ley Valenciana de Servicios Sociales.

En primer lugar, entre las ventajas que podemos enunciar, la participación ciudadana en la gestión pública es sinónimo de garantía de legitimidad en la toma de decisiones. Estas, no pueden limitarse a contemplar un desideratum de voluntades partidistas, sino que han de ser expresión de la vox populi, es decir, la adopción de decisiones más acordes con "las necesidades de la población a quien dirigen y representan, población a quien no tienen en cuenta a la hora de dirigir sus actuaciones" (Heras i Trias, 2008, p.19). "Reforzar las capacidades y competencias de esos gobiernos como la mejor garantía para democratizar y afianzar la toma de decisiones y mejorar la calidad de vida de los ciudadanos" (Subirats, 2016, p. 9). Tal y como destacan Navarro Gómez y Alba (2018), la participación ciudadana en los asuntos públicos favorece la reducción de la desigualdad y la exclusión de determinados colectivos poblacionales. Asimismo, la participación de las personas, bien a título individual como colectivo, así como de las entidades con y sin ánimo de lucro, es ejemplo de garantía democrática, tal y como enuncia el artículo 9.2 de la Constitución Española: "facilitar la participación de todos los ciudadanos en la vida política, económica, cultural y social". De acuerdo con Delgado Barrio (1993, p. 2318), ello se ha convertido en la jurisprudencia en un fundamento sobre el que legitimar el principio de participación concluyendo que: "corresponde a los poderes públicos (...), utilizar al máximo las posibilidades que el ordenamiento jurídico ofrece a los ciudadanos para participar". Desde el punto de vista ciudadano, el fomento de mecanismos de participación ciudadana por los poderes públicos puede traducirse en un cambio o reconsideración de las políticas públicas. Además de ello, el fomento de mecanismos transversales de participación ciudadana por los poderes públicos implica un mayor compromiso, conocimiento y apropiación de aquello que se considera como público3: "que la gente tenga protagonismo y que sea sujeto de decisiones; (...) del hacer tuyo, asumir, comprometerse: apoderarse o, como se ha inventado, empoderarse" (Heras i Trias, 2008, p. 23). A esta idea subyacente del empowerment en la acción política, cabe añadirle el concepto de "coproducción de políticas públicas", puesto que esta "apropiación" alude directamente a un "nuevo modo de abordar las políticas públicas a partir de la implicación (participación) de la ciudadanía orientada a la implementación de soluciones, una nueva forma de trabajar y de relacionarse" (Parés, 2017, p. 26).

En lo relativo a las dificultades, desde un punto de vista de los poderes públicos, la existencia de pluralidad de intereses y demandas no ha de responder necesariamente a un interés ciudadano unitario. El proceso de conversión entre interés, necesidad y demanda, en "problema público" implica el reconocimiento institucional en la agenda política, constituyéndose como la: "respuesta del sistema político-administrativo a una situación de la realidad social juzgada políticamente como inaceptable" (Subirats, et al., 2008, p. 33). Ante este hecho, las necesidades actuales no únicamente exigen contar con los instrumentos y las herramientas adecuadas para hacerles frente, sino que también cabe considerar de qué deben ocuparse los poderes públicos al objeto de obtener resultados más eficaces y eficientes (Subirats, 2006). Además, la articulación e implementación a posteriori de mecanismos de participación, implica apostar por instrumentos accesibles y legítimos, y por el esfuerzo de la accountability o rendición de cuentas a la comunidad. La rendición de cuentas, entendida como la transmisión de información (cuantitativa y/o cualitativa), así como a la justificación de las actuaciones políticas llevadas a cabo en los términos adecuados para la comprensión de las mismas por parte de la ciudadanía (Pereira y Jaráiz, 2015).

En lo relativo a los retos de futuro de los procesos participativos en la gestión pública, en la línea de Pintaudi, (2005): "podemos evaluar cuánto es difícil e insondable el ejercicio del poder por el pueblo, porque comprender y defender el interés público, de la mayoría, cuando no existe más un esprit de corps es muy difícil". Además de ello, el funcionamiento democrático multinivel (estatal, regional y local) de los poderes públicos obliga a disponer de mecanismos de participación ciudadana más allá del sufragio universal, y a que dicha participación haya de 
extenderse a "todos los procesos decisionales públicos, estableciendo cauces de información, transparencia, comunicación y control de los ciudadanos respecto a los poderes públicos (democracia participativa), concretándose así los derechos de participación y/o de buena administración" (Martín Núñez, 2013, p. 114).

Por todo lo expuesto brevemente, en los epígrafes siguientes se detalla el proceso participativo para la elaboración de la Ley Valenciana de Servicios Sociales, profundizando especialmente en el diseño de los mecanismos e instrumentos de participación por parte de la Vicepresidencia i Conselleria d'Igualtat i Polítiques Inclusives, y en el papel protagónico de la participación de los agentes sociales a lo largo de todo el proceso normativo.

\section{El punto de partida en la Comunitat Valenciana: los antecedentes al nuevo modelo social valenciano}

Para ahondar en la situación de la Comunitat Valenciana, en primer lugar, según el último Índice DEC (2018), tiene un desarrollo irrelevante, junto con Murcia, Andalucía, Madrid y Canarias, coincidiendo además con aquellas CC. $\mathrm{AA}^{4}$ con leyes de segunda generación. De igual modo, las CC. AA con mayor trayectoria en el desarrollo y reconocimiento legislativo de los servicios sociales como derecho subjetivo, coincide con aquellas que ocupan las primeras posiciones en el Índice DEC tales como el País Vasco y la Comunidad Foral de Navarra.

Gráfico 1. Índice DEC por Comunidades Autónomas.

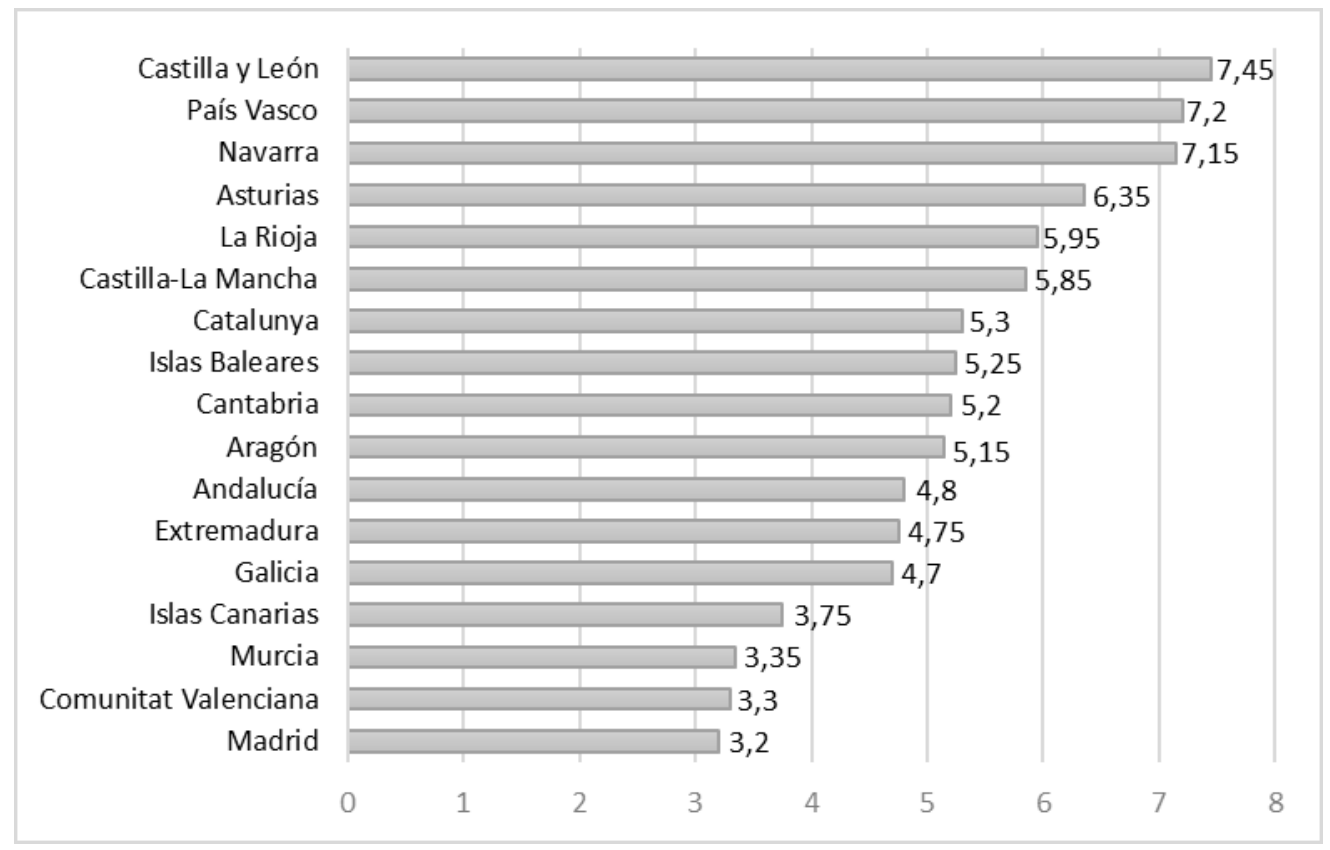

Fuente: Elaboración propia a partir de Índice DEC (2018, p. 20).

En el caso que nos ocupa, la ausencia de planificación, desarrollo y financiación en la Comunitat Valenciana ha ido hacia una creciente y notable desigualdad territorial, no únicamente con respecto a las demás comunidades autónomas, sino también en detrimento de los propios municipios de la región. El fortalecimiento del espacio local como aquel donde se desarrolla la vida cotidiana y se expresa la dicotomía entre inclusión-exclusión, es donde se produce la cobertura de las necesidades básicas y, en este sentido, las políticas sociales de proximidad han adquirido un gran protagonismo en el desarrollo -en la medida

\footnotetext{
Posterior a la publicación del Informe DEC (2018), Canarias y la Comunitat Valenciana en el año 2019 aprobaron leyes de tercera generación en el ámbito de los servicios sociales: Ley 16/2019, de 2 de mayo, de Servicios Sociales de Canarias y la Ley 3/2019, de 18 de febrero, de la Generalitat, de Servicios Sociales Inclusivos de la Comunitat Valenciana.
} 
de sus posibilidades-de los servicios sociales. Desde la aprobación de la Ley 5/1997, de 25 de junio, de la Generalitat, por la que se regula el Sistema de Servicios Sociales de la Comunitat Valenciana, el desarrollo normativo ha sido inexistente e incluso dicha ley ha estado sustentada sobre normativa-Mapeo de los Servicios Sociales de la Comunitat Valenciana (1991)- basada en la Ley 5/1989, de 6 de julio, de Servicios sociales de la Comunidad Valenciana. Ante ello, desde el año 2005, el Síndic de Greuges informó de la situación de emergencia en materia de servicios sociales, a través de numerosos informes, sobre: salud mental, violencia de género, dependencia, atención temprana, renta garantizada de ciudadanía, servicios sociales generales, menores tutelados, pobreza y exclusión social en los municipios valencianos, entre otros. En particular, el mismo Síndic de Greuges realizó varias recomendaciones en sus informes -Situación de los Servicios Sociales Valencianos (2013) y El papel de los servicios sociales generales en la lucha contra la pobreza y la exclusión social en los municipios valencianos durante la crisis" (2017)- y aboga por la actualización de la Ley 5/97, la apuesta por el derecho subjetivo y un Catálogo de Prestaciones, la garantía de la estabilidad presupuestaria en la financiación, organización funcional y territorial... (Martínez-Martínez, 2017).

De acuerdo con Uceda-Maza y Martínez-Martínez (2015), el gobierno autonómico en las dos últimas décadas "se ha desinhibido de la Red Básica de Prestaciones Sociales, trasladando la necesidad de financiación de la misma a la voluntad y a la posibilidad de las Corporaciones Locales" (citado en Martínez-Martínez, 2017, p. 110). Tal y como expone ampliamente Martínez-Martínez en su tesis doctoral, la situación de la Comunitat Valenciana se ha caracterizado en la geografía española por el abandono de los derechos sociales, especialmente, en el ámbito de los servicios sociales.

Las nuevas necesidades sociales que han emergido en las últimas décadas, requieren políticas públicas que se ajusten a la realidad de la sociedad valenciana. Ante ello, el Acuerdo del Botánico (2015) "marcó como aspiración política avanzar en las políticas igualdad, de derechos sociales universales y de sociedad inclusiva, enfatizando sobre la garantía de protección social de las personas" (Uceda-Maza, Martínez-Martínez y Caravantes, 2018, p. $315)$.

\section{El proceso participativo de la Ley Valenciana de Servicios Sociales}

Bajo la premisa del primer eje del Acuerdo del Botánico $(2015)^{5}$-“el rescate a las personas"la Generalitat Valenciana manifestó su compromiso de garantizar que "toda persona debe tener cubiertas las necesidades vitales básicas. Todos los valencianos tienen derecho a vivir en condiciones que garanticen su dignidad como seres humanos" (p. 2). Para llevar a cabo tal compromiso, desde el año 2015, la Vicepresidència i Conselleria d'Igualtat i Polítiques Inclusives apostó por contar con profesionales de la disciplina del Trabajo Social de reconocida trayectoria profesional en el ámbito de los servicios sociales para la construcción del nuevo modelo social valenciano (Uceda-Maza, 2017). Por su parte, el Gobierno del Botánico $\mathrm{II}^{6}$ (2019) ha mantenido al frente del equipo directivo de la Vicepresidencia y Políticas Inclusivas a quienes ya ostentaban cargos de alta responsabilidad e incluso, el mencionado equipo ha sido reforzado, incorporando a un trabajador social al frente de la Dirección General Institut Valencià de Formació, Innovació i Qualitat dels Serveis Socials (IVAFIQ).

Especialmente, el desarrollo de la Ley 3/2019, de 18 de febrero, de la Generalitat, de Servicios Sociales Inclusivos de la Comunitat Valenciana fue impulsado por la Delegación del Consell para el Modelo Social Valenciano, a través del diseño de una metodología participativa con los actores sociales al objeto de hacer efectivo el derecho de participación de la comunidad. En primera instancia, el encargo institucional respondía a articular un verdadero Sistema Público Valenciano de Servicios Sociales con la finalidad de garantizar el derecho subjetivo, con el compromiso autonómico de hacer frente a las nuevas necesidades sociales y bajo el principio de responsabilidad pública. Para ello, se diseñaron las cuatro etapas detalladas a continuación:

\footnotetext{
"Nombre que recibió el pacto programático del gobierno de la novena legislatura de la Comunitat Valenciana, firmado por el PSPV-PSOE, Compromís y Podemos el 11 de junio de 2015” (Uceda-Maza, Martínez-Martínez y Caravantes, 2018, p. 315).

6 Denominación con la que se conoce la Administración de la Generalitat en la X Legislatura.
} 
Esquema 1. Etapas de elaboración de la Ley de Servicios Sociales de la Comunitat Valenciana.

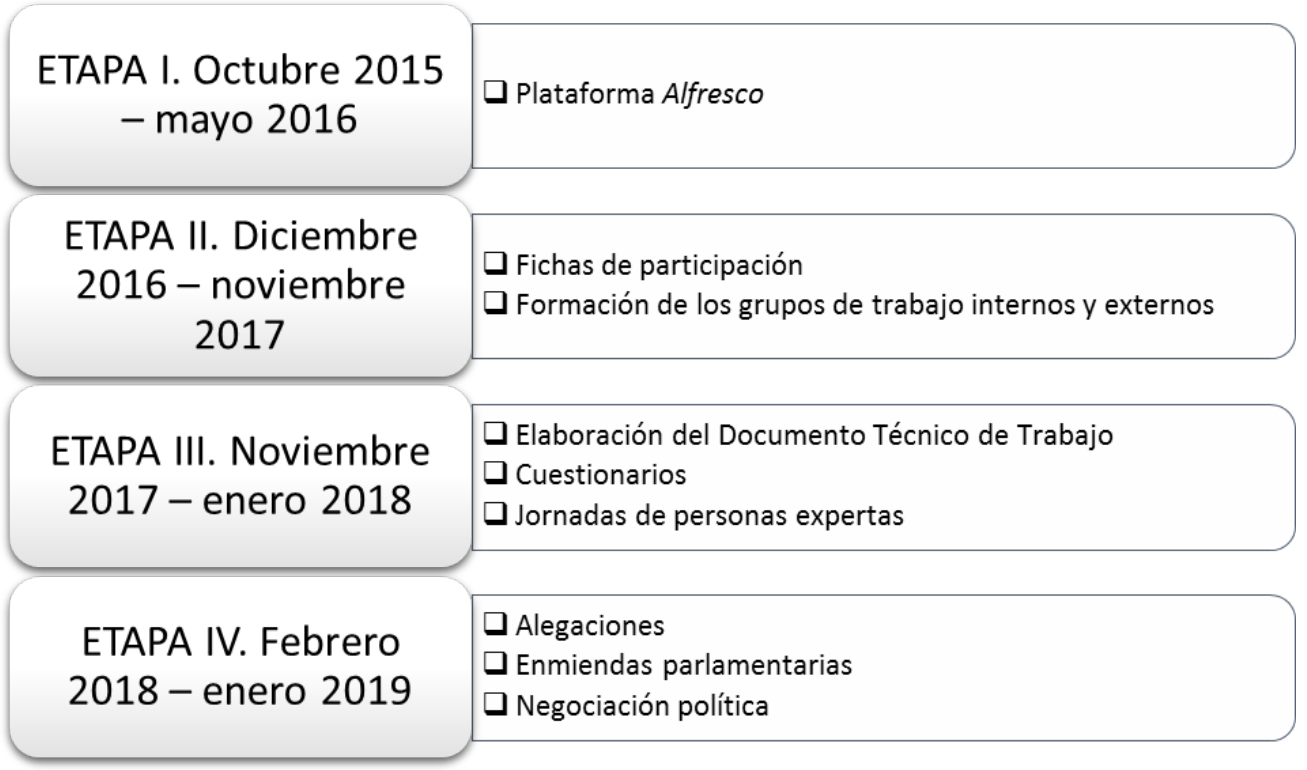

Fuente: Elaboración propia.

\subsection{Primera etapa: de octubre 2015 a mayo} de 2016

El proyecto normativo de servicios sociales fue iniciado desde la Dirección General de Planificación a través de una plataforma digital de la Generalitat Valenciana de consulta abierta a toda la ciudadanía. Tal y como indica el propio informe de participación de la norma que nos ocupa, en esta primera fase la participación se extendió a cerca de un centenar de personas, entre las que se identifican personas que son o han sido usuarias de servicios sociales, bien directamente o como familiares de las mismas, así como profesionales del ámbito de servicios sociales, sanidad, educación, entidades del tercer sector de acción social, sindicatos, universidades, etc. A pesar de que el número de participantes no era muy extenso, la consulta sí recoge un número significativo de aportaciones desde diferentes ángulos, tal y como se presenta a continuación:

1. Derechos de la ciudadanía en el sistema de servicios sociales: servicios sociales concebidos como derecho subjetivo, derecho a la participación, deberes profesionales y de las personas usuarias...

2. Medidas para garantizar los mencionados derechos: aprobación de una nueva
Ley, financiación acorde a las necesidades sociales de la ciudadanía, mejora en la gestión, coordinación, inspección y supervisión...

3. Administraciones gestoras de servicios sociales: gestión conjunta (Generalitat Valenciana, Diputaciones Provinciales y Ayuntamientos) versus gestión exclusivamente autonómica/local...

4. Órganos de participación y tipo de participación en el sistema de servicios sociales: órganos de participación permanente versus participación preceptiva, participación como derecho y como deber de las personas profesionales...

5. Coordinación con otros sistemas de protección social y tipología de la misma (interdepartamental, interadministrativa y técnica): coordinación funcional y eficaz, trabajo en red, planificación, seguimiento y evaluación conjunta...

6. Sugerencias: mejorar las ratios de personal, financiación....

El compendio de las aportaciones fue empleado para el diseño y planificación de la segunda etapa. 


\subsection{Segunda etapa: de diciembre de 2016 a noviembre de 2017}

Partiendo de la pluralidad y diversidad de información obtenida en la primera fase, en la Delegación del Consell para el Modelo Social Valenciano se diseñaron un total de once fichas con descriptores que permitían una mayor profundización sobre los ítems abordados en la plataforma digital. En concreto, el contenido de las fichas recogía los grandes apartados que posteriormente estructurarían el cuerpo normativo de la ley tales como: principios, derechos y deberes, estructura te- rritorial, funcional y competencial, catálogo de prestaciones, financiación, participación, etc. En este sentido, gracias a la colaboración de la Federación Valenciana de Municipios y Provincias (FVMP), las mencionadas fichas fueron enviadas a los 542 municipios de la Comunitat Valenciana -Castellón (135), Valencia (266) y Alicante (141)-, a sindicatos, colegios y asociaciones profesionales, universidades públicas valencianas, empresas y entidades que desarrollan su actuación en el ámbito de los servicios sociales. De todo ello, se obtuvieron un total de 418 fichas distribuidas de la siguiente forma:

Gráfico 2. Número de fichas aportadas por sector.

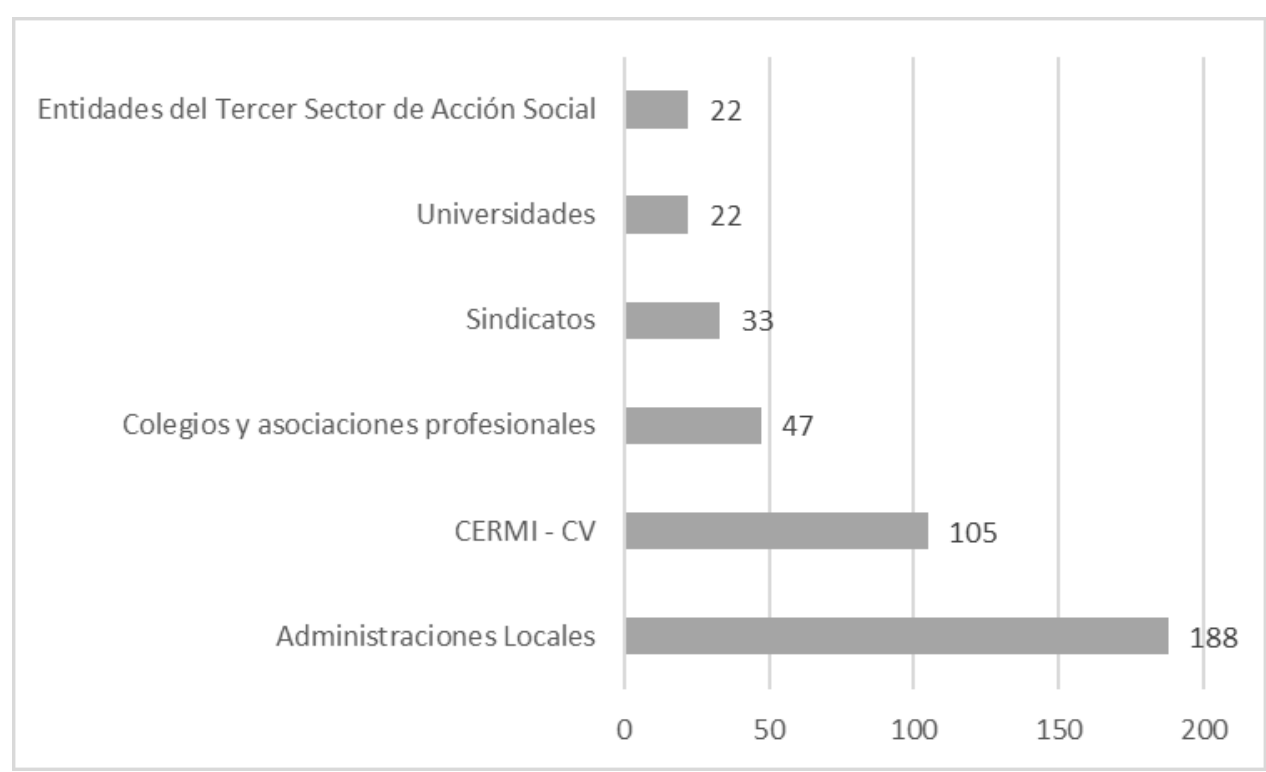

Fuente: Elaboración propia a partir de la Vicepresidència i Conselleria d'Igualtat i Polítiques Inclusives (2019).

Tal y como aparece reflejado en el gráfico 2, las Administraciones Locales y el CERMIComunitat Valenciana son quienes han presentado el grueso de las fichas (188 y 105 respectivamente), siguiéndole los colegios y las asociaciones profesionales (47), los sindicatos (33), las universidades (22) y las entidades del Tercer Sector de Acción Social (22).

Con los resultados obtenidos, se elaboró un Documento Técnico de Trabajo cuyo contenido recogía las principales aportaciones que la futura Ley de Servicios Sociales debía contener: derechos y deberes, principios, estructura territorial, funcional y competencial, catálogo de prestaciones, fuentes de financiación, participación social y participación de la iniciativa social y de la iniciativa privada entre otras cuestiones. Para su elaboración, se formaron distintos grupos de trabajo (tanto internos como externos a la conselleria):

a. Grupo motor (de carácter técnico): Formado por profesionales con amplia experiencia y conocimiento en el ámbito de los servicios sociales de las distintas Direcciones Generales de la Vicepresidencia i Conselleria d'Igualtat i Polítiques Inclusives, perso- 
nal de la $\mathrm{DCMSV}^{7}$, así como personal de la Subdirección General de Planificación, Ordenación, Evaluación y Calidad, del Servicio de Coordinación de Servicios Sociales Generales y del Servicio de Coordinación Sociosanitaria y Técnica de Tutelas.

2. Grupo interno (de carácter político): Formado por responsables políticos de la Vicepresidencia i Conselleria d'Igualtat i Polítiques Inclusives (Secretarias Autonómicas, Delegación del Consell para el Modelo Social Valenciano, Direcciones Generales y Direcciones Territoriales).

El primero de ellos fue el responsable de la redacción, impulso, promoción y análisis de la participación y posterior devolución de la información a los agentes sociales. La devolución del documento técnico de trabajo fue difundida entre diferentes grupos de trabajo externos al objeto de retroalimentar el propio texto. Tales grupos de trabajo se subdividieron en dos tipologías:

\section{i. Grupo de Administraciones Locales-} Federación Valenciana de Municipios y Provincias: representantes tanto del ámbito político como profesional de pequeños, medianos y grandes municipios, así como de las Diputaciones Provinciales.

ii. Grupo de personas expertas en Servicios Sociales: personas de reconocido prestigio y trayectoria profesional en el ámbito de los Servicios Sociales en España.

Además de tales grupos de trabajo, se constituyeron grupos de trabajo de consulta puntual-secuencial con los distintos actores sociales (sindicatos, colegios y asociaciones profesionales, entidades del tercer sector y empresas del ámbito social).

En el terreno político, la Delegación del Consell para el Modelo Social Valenciano realizó un gran esfuerzo paralelo para la rendición de cuentas con el resto de partidos políticos, realizando distintas reuniones de interlocución en la Comisión de Política Social de Les Corts y en el Fórum de Participación. Asimismo, también se presentaron las bases del Documen- to Técnico de Trabajo, el proceso participativo y los avances producidos hasta el momento en distintos debates abiertos a la ciudadanía.

En último lugar, en la segunda etapa se creó un grupo de trabajo con las universidades públicas valencianas formado por profesorado que fue directamente designado por el Rectorado de las respectivas universidades.

\subsection{Tercera etapa: de noviembre de 2017 a enero de 2018}

En la tercera etapa, la estrategia seguida por la Delegación del Consell para el Modelo Social Valenciano fue profundizar en el contenido del documento técnico de trabajo para desarrollar posteriormente el anteproyecto de Ley de Servicios Sociales de la Comunitat Valenciana. Al objeto de clarificar conceptos y el propio contenido, se elaboró un cuestionario de 52 preguntas organizado en siete bloques temáticos (1. Título Preliminar; 2. Sistema Público Valenciano de Servicios Sociales; 3. Catálogo de Prestaciones y Carteras del Sistema Público Valenciano de Servicios Sociales; 4. Planificación, Ordenación e Intervención del Sistema Público Valenciano de Servicios Sociales; 5. Iniciativa Privada; 6. Participación; y 7. Financiación y Sostenibilidad del Sistema), con tres modelos de respuesta: abierta, binomial (adecuado/inadecuado) y escala Likert. Fue enviado a los grupos externos de trabajo conformados en la segunda fase. Paralelamente, se llevaron a cabo las Jornadas de Personas expertas en legislación de Servicios Sociales ${ }^{8}$ en la ciudad de Alzira en el mes de noviembre de 2017. En tales Jornadas fue utilizada una metodología participativa basada en distintos grupos de discusión, formados cada uno de ellos por las personas integrantes de los cuatro grupos de trabajo, con la finalidad de contrastar opiniones tanto de otras experiencias autonómicas en servicios sociales, de la práctica diaria de los municipios, así como de la propia gestión política. Para la organización de los distintos grupos de discusión se tuvo en consideración que su composición no superara los diez integrantes y que, a su vez, fuera plural y diversa la representación de los actores. Por cada grupo de discusión, se estableció una persona moderadora y una persona

Integrado por profesionales de las siguientes disciplinas: Derecho, Geografía, Economía, Trabajo Social, Sociología, Psicología y Ciencias Políticas.

Véase https://bit.ly/21MEsLJ 
que recogiera el conjunto de aportaciones surgidas en el debate. En tales jornadas, participaron en total 50 personas $(21$ hombres y 29 mujeres), de los cuales el 10\% eran personas expertas de otras Comunidades Autónomas y el $90 \%$ profesionales y responsables políticos de la Comunitat Valenciana. En base a ello, las temáticas tratadas y los resultados obtenidos en cada uno de los grupos de trabajo fueron los siguientes:

Cuadro 1. Resultados obtenidos en las Jornadas de Personas expertas en legislación de Servicios Sociales

\begin{tabular}{|c|c|c|c|}
\hline & PROPUESTAS DE MEJORA & $\begin{array}{c}\text { DUDAS Y } \\
\text { COMENTARIOS }\end{array}$ & $\begin{array}{l}\text { ASPECTOS } \\
\text { POSITIVOS }\end{array}$ \\
\hline $\begin{array}{l}\text { Objeto y princi- } \\
\text { pios de los Ser- } \\
\text { vicios Sociales. } \\
\text { Sistema Público } \\
\text { Valenciano de Ser- } \\
\text { vicios Sociales y } \\
\text { Servicios Sociales } \\
\text { Valencianos. Dere- } \\
\text { chos y Compromi- } \\
\text { sos. Participación } \\
\text { cívica }\end{array}$ & $\begin{array}{l}\text { Clarificación del objeto } \\
\text { Inclusión de nuevos principios: justicia social, accesi- } \\
\text { bilidad universal, perspectiva de género... } \\
\text { Inclusión de nuevos derechos de las personas usuarias: } \\
\text { participación en su proceso de intervención, un segun- } \\
\text { do diagnóstico... } \\
\text { Inclusión de nuevos compromisos de las personas } \\
\text { usuarias: responsabilidad de las mismas en la promo- } \\
\text { ción de su propio cambio, ser veraces y asistir a las } \\
\text { entrevistas... } \\
\text { Inclusión de nuevos derechos de las personas profesio- } \\
\text { nales: supervisión profesional, disponer de espacios de } \\
\text { coordinación ... } \\
\text { Inclusión de nuevos compromisos de las personas } \\
\text { profesionales: obligación de poner en conocimiento de } \\
\text { la autoridad competente una mala praxis profesional y } \\
\text { vulneración de derechos... }\end{array}$ & $\begin{array}{l}\text { Enfoque munici- } \\
\text { palista ¿excesivo } \\
\text { peso a los ayunta- } \\
\text { mientos? } \\
\text { Escasa alusión al } \\
\text { nivel comunitario } \\
\text { Arriesgado garan- } \\
\text { tizar una respuesta } \\
\text { inmediata en casos } \\
\text { de urgencia }\end{array}$ & $\begin{array}{l}\text { Protagonismo de los mu- } \\
\text { nicipios } \\
\text { Confidencialidad como } \\
\text { fortaleza del proceso de } \\
\text { intervención }\end{array}$ \\
\hline $\begin{array}{l}\text { Estructura funcio- } \\
\text { nal, territorial y } \\
\text { competencial del } \\
\text { Sistema Público } \\
\text { Valenciano de } \\
\text { Servicios Sociales. } \\
\text { Financiación. }\end{array}$ & $\begin{array}{l}\text { Nueva reestructuración. Especificar las funciones de } \\
\text { cada nivel } \\
\text { Mayor hincapié en la función de intervención de la } \\
\text { Atención Primaria Básica } \\
\text { Las agrupaciones de municipios no pueden ser de } \\
\text { carácter voluntario } \\
\text { Determinar protocolos de coordinación entre las Admi- } \\
\text { nistraciones competentes } \\
\text { Establecer mínimos de financiación por ley y desa- } \\
\text { rrollarlo con mayor profundidad en una normativa } \\
\text { posterior } \\
\text { Equilibrar la financiación de los ayuntamientos en } \\
\text { función de su capacidad económica }\end{array}$ & $\begin{array}{l}\text { La creación de un } \\
\text { nivel intermedio } \\
\text { continúa orientan- } \\
\text { do la intervención } \\
\text { por colectivos en } \\
\text { lugar de por nece- } \\
\text { sidades } \\
\text { Compleja distribu- } \\
\text { ción competencial } \\
\text { y financiera }\end{array}$ & $\begin{array}{l}\text { Importante cambio en el } \\
\text { modelo territorial } \\
\text { El principio de cohesión } \\
\text { territorial es clave } \\
\text { Positivo establecer me- } \\
\text { didas de discriminación } \\
\text { positiva en la financiación } \\
\text { de los equipos de los } \\
\text { espacios vulnerables }\end{array}$ \\
\hline $\begin{array}{l}\text { Metodología de } \\
\text { intervención, coor- } \\
\text { dinación, compo- } \\
\text { sición de equipos } \\
\text { profesionales, } \\
\text { personal de refe- } \\
\text { rencia y ratios. }\end{array}$ & $\begin{array}{l}\text { Incluir la figura del/la asesor/a jurídico/a en la Aten- } \\
\text { ción Primaria Básica } \\
\text { Propuesta de constitución de equipos de emergencia y } \\
\text { urgencia social } \\
\text { Mejorar la definición de trabajo en red } \\
\text { Definir los procedimientos y procesos de intervención } \\
\text { social }\end{array}$ & $\begin{array}{l}\text { Incertidumbre } \\
\text { respecto a la po- } \\
\text { sibilidad de con- } \\
\text { siderar desde los } \\
\text { municipios la ratio } \\
\text { mínima como ratio } \\
\text { máxima }\end{array}$ & $\begin{array}{l}\text { Fijación por ley de los } \\
\text { perfiles profesionales de } \\
\text { los equipos } \\
\text { Diferencia entre equipo } \\
\text { de intervención social y } \\
\text { equipo de apoyo adminis- } \\
\text { trativo } \\
\text { Trabajador/a social como } \\
\text { profesional de referencia } \\
\text { Interdisciplinariedad de } \\
\text { los equipos }\end{array}$ \\
\hline
\end{tabular}




\begin{tabular}{|c|c|c|c|}
\hline & PROPUESTAS DE MEJORA & $\begin{array}{c}\text { DUDAS Y } \\
\text { COMENTARIOS }\end{array}$ & $\begin{array}{l}\text { ASPECTOS } \\
\text { POSITIVOS }\end{array}$ \\
\hline $\begin{array}{l}\text { Planificación In- } \\
\text { vestigación, inno- } \\
\text { vación y calidad } \\
\text { Formación. }\end{array}$ & $\begin{array}{l}\text { Cambiar las estrategias de planificación a nivel de Área } \\
\text { de Servicios Sociales } \\
\text { Cambiar los plazos establecidos del Plan Estratégico: } \\
\text { mínimo cuatro años y máximo seis }\end{array}$ & $\begin{array}{l}\text { Incertidumbre } \\
\text { acerca de la } \\
\text { existencia de fi- } \\
\text { nanciación para } \\
\text { el desarrollo de la } \\
\text { planificación }\end{array}$ & $\begin{array}{l}\text { Incorporación del Sis- } \\
\text { tema de Información } \\
\text { Multiterritorial }\end{array}$ \\
\hline $\begin{array}{l}\text { Colaboración con } \\
\text { la iniciativa pri- } \\
\text { vada Catálogo de } \\
\text { Prestaciones del } \\
\text { Sistema Público } \\
\text { Valenciano de Ser- } \\
\text { vicios Sociales }\end{array}$ & $\begin{array}{l}\text { Cambiar el término "prestaciones técnicas" por "pres- } \\
\text { taciones profesionales" } \\
\text { Incluir la prestación de "alojamiento alternativo" } \\
\text { Incluir la tipología de 'prestaciones de servicios" }\end{array}$ & $\begin{array}{l}\text { Se deben garanti- } \\
\text { zar todas las pres- } \\
\text { taciones técnicas } \\
\text { Organizar las pres- } \\
\text { taciones por nive- } \\
\text { les funcionales }\end{array}$ & $\begin{array}{l}\text { Establecer un Catálogo de } \\
\text { mínimos } \\
\text { Fijación por ley de las } \\
\text { prestaciones garantizadas } \\
\text { Denominaciones propias }\end{array}$ \\
\hline
\end{tabular}

Fuente: Elaboración propia a partir de Caravantes, Uceda-Maza y Martínez-Martínez (2018).

Con el conjunto de los resultados aportados en los cuestionarios, junto con la información extraída en el desarrollo de las mencionadas Jornadas, en la Delegación del Consell para el Modelo Social Valenciano se reelaboró el documento técnico de trabajo, transformándolo en el anteproyecto de Ley de Servicios Sociales que fue presentado a la ciudadanía el 31 de enero de 2018, a cuyo acto de presentación asistieron más de mil personas de toda la Comunitat Valenciana9.

\subsection{Cuarta etapa: de enero de 2018 a febre- ro de 2019}

Posterior a la presentación pública del anteproyecto de Ley, se inició el proceso de alega- ciones, de conformidad con lo dispuesto en el artículo 133.2 de la Ley 39/2015, de 1 de octubre, del Procedimiento Administrativo Común de las Administraciones Públicas. En dicho proceso, se recibió un total de 411 alegaciones relativas a personas físicas (75), entidades del tercer sector (85), colegios y asociaciones profesionales (35), sindicatos (76), Entidades Locales y FVMP (35), Universidades (7) y empresas (98). Respecto a su valoración, cabe destacar que la gran totalidad fueron estimadas o estimadas parcialmente y su contenido fue "incorporado al texto del anteproyecto en el $58,75 \%$ de los casos, ya sea recogiendo las aportaciones en su totalidad o parte de las mismas" (Vicepresidencia i Conselleria d'Igualtat i Polítiques Inclusives, 2018, p. 5):

Gráfico 3. Tipo de respuesta a las alegaciones presentadas.

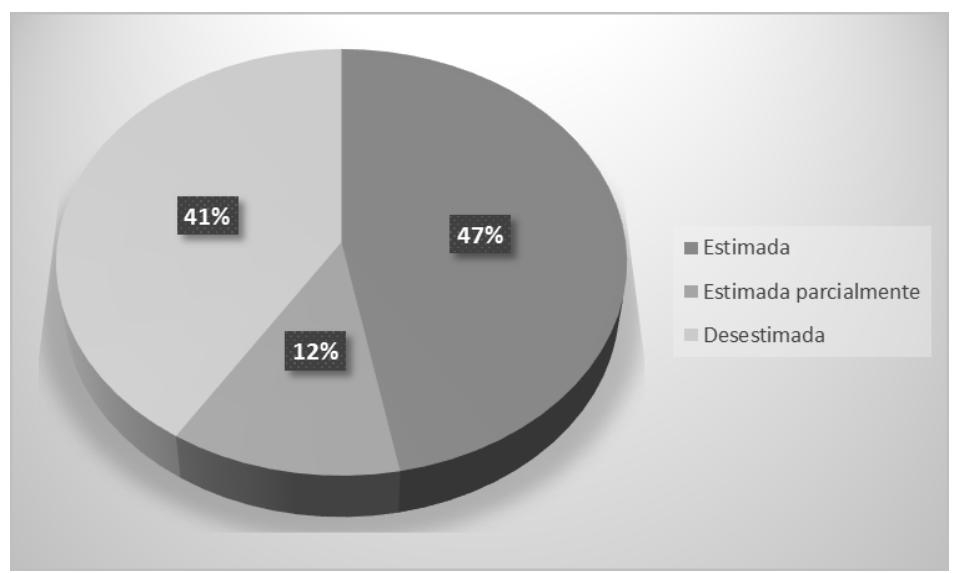

Fuente: Elaboración propia a partir de Vicepresidencia i Conselleria d'Igualtat i Polítiques Inclusives (2018).

$9 \quad$ Ante la elevada expectación ciudadana, fue necesario habilitar una sala anexa para la visualización del acto por parte de todos y todas las asistentes https://bit.ly/2rx896J 
Además de ello, en el siguiente gráfico 4 están reflejadas las valoraciones realizadas a cada una de las alegaciones presentadas, en función del sector. En este sentido, cabe destacar que en la gran totalidad de los sectores las alegaciones han sido valoradas como "es- timadas" o "estimadas parcialmente", a excepción del contenido de las alegaciones procedentes de personas físicas y de las empresas con ánimo de lucro, cuyas valoraciones en ambos casos han sido mayoritariamente desestimatorias.

Gráfico 4. Valoración de las alegaciones en función del sector.

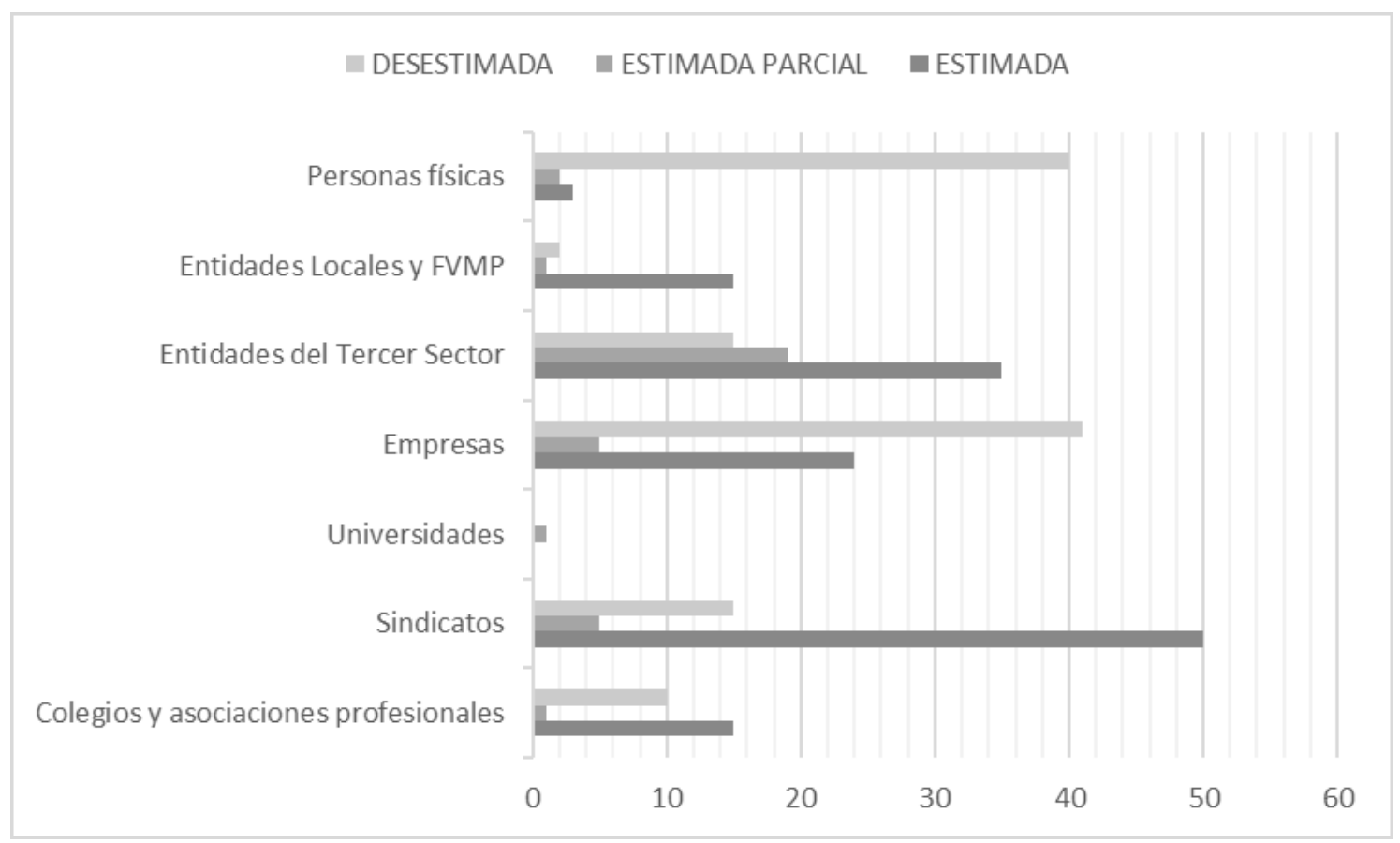

Fuente: Elaboración propia a partir de Vicepresidencia i Conselleria d'Igualtat i Polítiques Inclusives (2018).

Posteriormente a las alegaciones, se elaboró un informe pormenorizado en el que se detallaba, la justificación de la valoración realizada desde la Delegación del Consell para el Modelo Social Valenciano. Tal informe, de carácter cuantitativo y cualitativo, fue enviado al conjunto de entidades e instituciones participantes en la fase de alegaciones. Además del conjunto de alegaciones de las entidades, también se elaboró un informe sobre el tipo de respuesta a las 37 alegaciones presentadas por las distintas Consellerias de la Generalitat, de las cuales fue estimado el $83,78 \%$, el $10,81 \%$ estimadas parcialmente y el $5,40 \%$ desestimadas. La distribución de las distintas alegaciones presentadas por las Consellerias se describe en el siguiente gráfico: 
Gráfico 5. Número de alegaciones según tipo de respuesta y organismo.

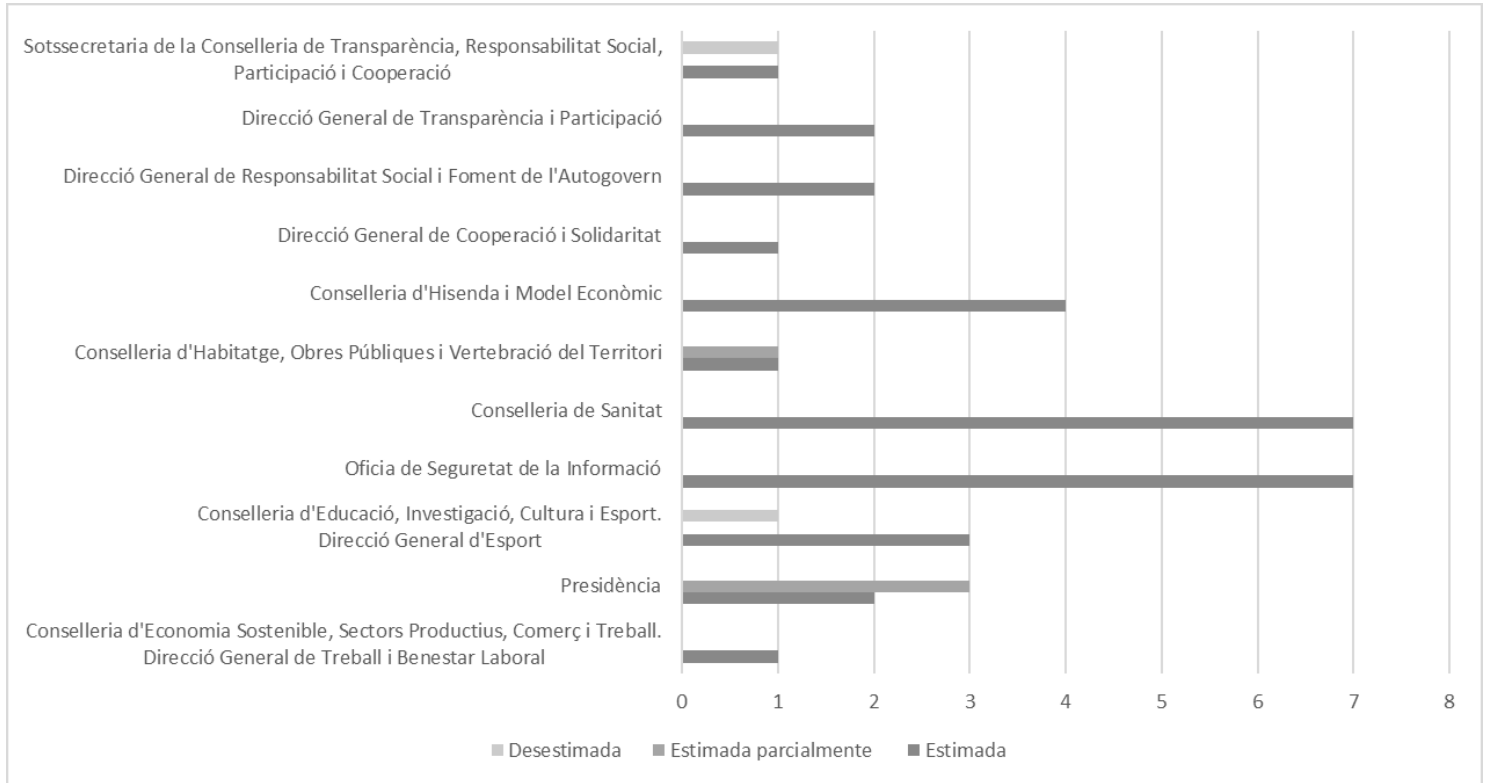

Fuente: Elaboración propia. Seguidamente, se continuó con los informes preceptivos fijados según el procedimiento que entraña una normativa tales como: informe de la abogacía de la Generalitat, informe

del Comitè Econòmic i Social, informe del Consell Jurídic i Consultiu, memoria económica...

Posterior al visto bueno de tales informes por parte de los organismos competentes, el anteproyecto de Ley fue publicado en el Boletín Oficial de Les Corts (BOCV) como Proyecto de Ley de Servicios Sociales Inclusivos de la Comunitat Valenciana. A partir de su publicación, comenzó el proceso de elaboración de enmiendas entre los distintos partidos políticos con representación en Les Corts. Para su negociación, la Delegación del Consell para el Modelo Social Valenciano junto con la diputada por Castellón del grupo parlamentario Compromís mantuvieron reuniones paralelas con cada uno de los partidos políticos y no adscritos, con la finalidad de conocer las distintas posturas y argumentos al respecto. Finalmente, la Ley 3/2019, de 18 de febrero, de la Generalitat, de Servicios Sociales Inclusivos fue aprobada con 51 votos a favor, 30 votos en contra y 8 abstenciones.

\section{El derecho de participación ciudadana en los servicios sociales: una cuestión a desarrollar}

El desarrollo de la Ley 3/2019 haciendo partícipe a la comunidad (Canto Chac, 2008; Subirats, 2016), por un lado, supone legitimar la acción política (Heras i Trias (2008) en materia de servicios sociales $y$, por otro lado, garantizar el derecho de participación ciudadana (Delgado Barrio, 1993).

El extenso y amplio proceso de participación de la Ley 3/2019 se ha convertido, por un lado, en una iniciativa común, compartida y plural entre todos los actores sociales, haciendo de ella un proyecto de sociedad y creando un precedente para las posteriores iniciativas políticas de carácter social; y, por otro lado, ha constituido la respuesta del sistema político-administrativo a la crítica situación en la que se encuentran los servicios sociales en la Comunitat Valenciana. Resituar a la comunidad en el centro de la acción política significa revalorizar los principios democráticos ya que supone el espacio donde convergen no únicamente ciudadanía y necesidades, sino también el fundamento de la cohesión social (Martínez-Martínez, 2017).

El interés común por consolidar una legislación que amplíe los derechos de ciudadanía, conjuga la voluntad de la Administración y de la ciudadanía por conducir la política como un proceso y como un medio para la consecución de objetivos compartidos con la comunidad, ya lo que verdaderamente importante es "el contenido de las políticas públicas y no la asig- 
nación de responsabilidades" (Subirats, 2016, p. 103). En lo relativo a la accountability, la devolución de información a la ciudadanía (Pereira y Jaráiz, 2015) es parte del proceso de las nuevas formas de gobernanza, donde se produce una doble exigencia: 1) la de rendir cuentas a la ciudadanía por parte de los poderes públicos en un formato accesible y 2) la exigencia que la propia ciudadanía puede establecer ante los poderes públicos como resultado del proceso de participación.

Asimismo, los procesos de participación no están exentos de dificultades y retos de futuro. Una de las grandes dificultades, a lo largo de la elaboración de la Ley 3/2019, fue el fomento de la participación de las personas usuarias o familiares de estas (Pintaudi, 2005), tanto en el inicio como en las subsiguientes etapas. Incorporar a las bases sociales y a los sectores informales de nuestra sociedad en los procesos participativos, es además de una dificultad (tanto para la ciudadanía como para la Administración), un reto de futuro y una asignatura pendiente para la gobernanza multinivel (Martín Núñez, 2013).

\section{Conclusiones principales}

Por todo lo expuesto anteriormente, la atención de las necesidades sociales en la agenda política valenciana se traduce en situar a la comunidad en el centro de la acción política y la inversión en servicios sociales no implica únicamente una apuesta por la satisfacción de las necesidades de colectivos vulnerables, sino el compromiso por garantizar el bienestar de la sociedad en su conjunto. Tal y como se ha relatado, el caso valenciano evidencia que las nuevas formas de gobernanza no son antagónicas al sistema neoliberal, sino que tal y como se ha venido desarrollando, los modelos de de- mocracia participativa se han integrado en dicho sistema. Apostar por situar a la comunidad en el centro de la acción política, es sinónimo de garantizar el significado de demos krátos y el derecho de participación ciudadana en los asuntos públicos. No obstante, a pesar de que el derecho de participación es intrínseco a las nuevas formas de gobernanza propias de los regímenes políticos de los Estados democráticos, no es el único elemento para consolidar políticas públicas eficaces y eficientes. La participación ejerce un rol fundamental en las nuevas formas de gobernanza y en el ejercicio de la gobernabilidad, pero a ello es necesario agregar la apuesta por el desarrollo y por los derechos de ciudadanía. El derecho de participación en el ámbito de los servicios sociales contribuye a la consolidación de los derechos sociales, ciertamente amenazados por algunos partidos políticos.

Por su parte, los mecanismos de participación no son obligatorios, sino que la consideración de reconocer a la ciudadanía en el compendio de una política pública depende en buena medida de la voluntad de los poderes políticos. No obstante, cabe agregar que tales mecanismos frenan el distanciamiento de la equívoca convicción de los poderes públicos de desarrollar políticas adecuadas a las necesidades ciudadanas sin contar con su participación, puesto que los actores sociales disponen de un conocimiento crítico de la realidad social de las personas.

Gobernar implica no únicamente responder a la diversidad de problemáticas que existen en un territorio determinado, sino que es necesario el reconocimiento de la pluralidad de actores que conforman la sociedad. Sin el reconocimiento de los actores sociales que conforman la extensa red de servicios sociales, es inviable una acción de gobierno con, en y para la comunidad.

\section{Referencias bibliográficas}

Arnáez Arce, V.M. (2012). La participación del cooperativismo en las políticas públicas. Boletín de la Asociación Internacional de Derecho Cooperativo, 46, 185-199.

Barranco, C. (2009). Discapacidad y Trabajo Social: una mirada sobre los enfoques y los derechos sociales. Revista de Treball Social de Cataluña, 186, 81- 94.

Berrios, P. y Zapata, L. (2015). Participación ciudadana y políticas públicas para garantizar los derechos humanos y erradicar la discriminación de las personas con discapacidad en el Distrito. Revista Espacios Transnacionales, 4, 48-60.

Canto Chac, M. (2008). Gobernanza y participación ciudadana en las políticas públicas frente al reto del desarrollo. Política y Cultura, 30, 9-37. 
Caravantes, G.M. (2018a). El derecho a la ciudad y el derecho a la vivienda: El análisis de ODS 11 desde la exclusión residencial en el Sur y en el Norte, Los Derechos Humanos y los Objetivos de Desarrollo Sostenible. Comunicación llevada a cabo en el III Congreso Internacional sobre Derechos Humanos, 25-26 de octubre. Ilustre Colegio de Abogados de Valencia, València, España.

Caravantes, G.M., Uceda-Maza, F.X. y Martínez-Martínez, L. (2018b). El proceso participativo para la elaboración de la nueva Ley de Servicios Sociales de la Comunitat Valenciana desde el Trabajo Social. Comunicación presentada en el VII Congreso Red de Estudios sobre Políticas Sociales, REPS, Políticas Sociales ante horizontes de incertidumbre y desigualdad. 4-5 de octubre. Zaragoza, España.

Farinós, J. (2011). Inteligencia Territorial para la planificación y la gobernanza democráticas: los observatorios de los territorios. Workshop Mendoza: Red Iberoamericana de Observación Territorial, V, 45-69.

Farinós, J. y Romero, J. (2008). La gobernanza como método para encarar los nuevos grandes retos territoriales y urbanos. Boletín de la Asociación de Geógrafos Españoles, 46, 5-9.

Garcés Sanagustín, A. (2011). La participación en los servicios sociales. Revista Aragonesa de Administración Pública, 38, 123-154.

Heras i Trias, P. (2008). La acción política desde la comunidad. Análisis y propuestas. Barcelona: Editorial GRAÓ.

Marchioni, M. (2007). Planificación social y organización de la comunidad. Alternativas avanzadas a la crisis. Madrid: Popular.

Martín Núñez, E. (2013). Los derechos de participación política y administrativa en la constitución y en los Estatutos de Autonomía: especial referencia al Estatuto de Autonomía de Cataluña. Chapecó, 14(3), 113-132.

Martínez-Martínez, L. (2017). Definición de las necesidades sociales para un nuevo desarrollo del Sistema de Servicios Sociales de la Comunidad Valenciana, desde una metodología participativa (Tesis Doctoral, Facultat de Ciències Socials, Universitat de València, València).

Pereira, M. y Jaráiz, E. (2015). El Nuevo Servicio Público (NSP), un paradigma para la construcción de nuevos modelos metodológicos para el análisis de la administración pública. RIPS, 14 (2), 73-94.

Pintaudi, S.M. (2005). Participación ciudadana en la gestión pública: los desafiós políticos. Scripta Nova, Revista Electrónica de Geografía y Ciencias Sociales, 194(102).

Subirats, J. (2016). El poder de lo próximo. Las virtudes del municipalismo. Barcelona, Catarata.

Subirats, J. (2006). Algunos puntos clave sobre evaluación de políticas públicas con especial referencia al caso de las políticas sociales. Ánfora: Revista Cientifica de la Universidad Autónoma de Manizales, 13(21), 54-76.

Subirats, J. et al. (2008). Análisis y gestión de políticas públicas. Revista de estudios políticos. 145, 1-178.

Uceda-Maza, F.X., Martínez-Martínez, L. y Caravantes, G.M. (2018). Trabajo social y acción política: la experiencia de la Comunitat Valenciana en tiempos de transformaciones sociales. En: C. Verde, A.I. Lima y E. Pastor (coords.), El Trabajo Social ante los Desafios del Siglo XXI desde una Perspectiva Iberoamericana (pp. 309-321). Navarra: Aranzadi.

Uceda-Maza, F.X. y Martínez-Martínez, L. (2015). Presente, pasado y futuro de los Servicios Sociales Municipales en la Comunitat Valenciana: diagnóstico y propuestas para una nueva época, TS Nova: Trabajo Social y Servicios Sociales, 11, 69-90.

Vicepresidencia i Conselleria d'Igualtat i Polítiques Inclusive. (2018). Informe de respuesta a las alegaciones presentadas al anteproyecto de Ley de Servicios Sociales de la Comunitat Valenciana. Recuperado de: https://bit.ly/2k7M9M7 (consultado el 09-09-2019).

\section{Referencias normativas}

Ley 16/2019, de 2 de mayo, de Servicios Sociales de Canarias

Ley 3/2019, de 18 de febrero, de la Generalitat, de Servicios Sociales Inclusivos de la Comunitat Valenciana Ley $2 / 2017$, de 3 de febrero, de la Generalitat, por la función social de la vivienda de la Comunitat Valenciana Ley 19/2017, de 20 de diciembre, de la Generalitat, de Renta Valenciana de Inclusión

Ley $3 / 2017$, de 3 de febrero, de la Generalitat, para paliar y reducir la pobreza energética en la Comunitat Valenciana

Decreto 62/2017, de 19 de mayo, del Consell, por el que se establece el procedimiento para reconocer el grado de dependencia a las personas y el acceso al sistema público de servicios y prestaciones económicas. 
Ley 39/2015, de 1 de octubre, del Procedimiento Administrativo Común de las Administraciones Públicas

Ley 39/2006, de 14 de diciembre, de Promoción de la Autonomía Personal y Atención a las personas en situación de Dependencia

Ley 5/1997, de 25 de junio, de la Generalitat, por la que se regula el Sistema de Servicios Sociales de la Comunitat Valenciana

Ley 5/1989, de 6 de julio, de Servicios sociales de la Comunidad Valenciana

\section{Fuentes documentales}

ONU- Centro de Derechos Humanos. (1995). Derechos Humanos y trabajo social. Manual para escuelas de servicio social y trabajadores sociales. Ginebra: Organización de las Naciones Unidas.

Síndic de Greuges. (2003). Informe Especial a las Cortes Valencianas sobre la situación de las personas que padecen enfermedades mentales y de sus familias. Alicante. Publicaciones del Síndic de Greuges

Síndic de Greuges. (2013). Situación de los Servicios Sociales Generales en la Comunidad Valenciana. Recuperado de: http://bit.ly/2dlEc1V

Síndic de Greuges (2014a). Informe memoria de actuación general del Síndic en 2013 a las Cortes Valencianas. Recuperado de: http://bit.ly/2mNwX2e

Síndic de Greuges. (2014b). Informe sobre la Renta Garantizada de ciudadanía. Recuperado de: http://bit. ly/203XJqG

Síndic de Greuges (2015). Informe memoria de actuación general del Síndic en 2014 a las Cortes Valencianas. Recuperado de: http://bit.ly/2nYEoWj

Síndic de Greuges. (2017a). El papel de los servicios sociales generales en la lucha contra la pobreza y la exclusión social en los municipios valencianos durante la crisis. Recuperado de: http://bit.ly/2lfSvHp

Síndic de Greuges. (2017b). El ejercicio de la tutela de menores por las administraciones públicas valencianas. Recuperado de: http://bit.ly/2odgVQV 